\title{
Intra-abdominal seminoma in undescended testis with retroperitoneal nodal metastasis: A case report
}

\author{
Ranjan Kumar Patel ${ }^{1}$, Hitesh Sarda ${ }^{2}$ \\ From Senior Resident, ${ }^{1}$ Department of Radiodiagnosis, ${ }^{2}$ Department of Surgery, Maulana Azad Medical College, New Delhi, India
}

\begin{abstract}
Undescended testis or cryptorchidism is the most common predisposing factor in the development of testicular germ cell tumors with seminoma being the most common type. Therefore, testicular malignancy should be ruled out in case of an abdominal mass in a cryptorchid patient. Here, we present a case of intra-abdominal seminoma transformation of the undescended right testis with retroperitoneal nodal metastasis in a 54-year-old male which was clinically manifested as a gradually enlarging intra-abdominal mass and dull aching lower abdominal pain. He was managed with surgical removal, followed by chemotherapy.
\end{abstract}

Key words: Cryptorchidism, Intra-abdominal seminoma, Retroperitoneal nodal metastasis, Seminoma

$\mathrm{C}$ ryptorchidism is the most common predisposing factor in the development of testicular germ cell tumors. There is a variable chance of malignant transformation, ranging from $3.5 \%$ to $14.5 \%$ [1]. The abdominal testis has the highest risk, about four times higher than that of an inguinal ectopic testis. Seminoma is the most common histological type in such conditions [2]. The testicular region should be thoroughly looked at while evaluating an intra-abdominal mass on imaging in a male patient. Careful clinical history and local examination are also very important to prevent misdiagnosis in such a scenario.

\section{CASE REPORT}

A 54-year-old male presented to the surgical outpatient department with complaints of progressive swelling and pain in the lower abdomen for 3 months. The pain was gradual onset, dull aching, and localized in nature without any radiation. On examination, the vitals were stable. There was a history of weight loss of about $3 \mathrm{~kg}$ over 2 months. Local examination revealed a firm, nontender mass in the hypogastrium, extending up to the umbilical region. The right scrotal sac was empty.

Hemogram, liver function, and renal function parameters were normal except for a low hemoglobin level of $9.3 \mathrm{~g} / \mathrm{dl}$. Serum lactate dehydrogenase (LDH) level was markedly elevated (1075 IU/L) (normal range: 100-190 IU/L). Serum alfa fetoprotein (AFP)

\section{Access this article online}

Received - 29 March 2021

Initial Review - 14 April 2021

Accepted - 17 April 2021

DOI: 10.32677/IJCR.2021.v07.i04.013 and beta-human chorionic gonadotropin levels were within the normal laboratory range.

Ultrasonography showed the absence of a right testicle in the scrotal sac and normal left testis. A large, complex, heterogeneously hypoechoic intra-abdominal mass lesion with solid vascular areas was seen (Fig. 1a). Anechoic necrotic areas with multiple echogenic foci were seen within the mass (Fig. 1b). Contrast-enhanced computed tomography (CECT) abdomen (portal venous phase) revealed absent right spermatic cord (Fig. 2a) with a large, welldefined, heterogeneously enhancing intra-abdominal mass lesion of size $8.5 \mathrm{~cm} \times 14.4 \mathrm{~cm} \times 11.6 \mathrm{~cm}$ with variable necrotic areas (Fig. $2 \mathrm{~b}$ and c). Focal peritoneal thickening was seen in the vicinity of the lesion, suggestive of peritoneal involvement. In addition, an enlarged homogenously enhancing aortocaval lymph node was also present, suggestive of retroperitoneal nodal metastasis (Fig. 2d).

On 18-fluorodeoxyglucose (FDG)-positron emission tomography scan, both the mass (Fig. 3a) and retroperitoneal lymph node showed avid FDG uptake (Fig. 3b). Based on the absent right testis with FDG avid heterogeneously enhancing mass and enlarged enhancing retroperitoneal lymph node, a possibility of intra-abdominal testicular malignancy was suspected. On laparotomy, a large intra-abdominal encapsulated mass lesion was found, showing multiple necrotic areas, and focal areas of calcification. Testis was not identified separately.

Histopathological section from the viable part of the lesion revealed rests of tumor cells separated by fibrous septae. The individual cells were polygonal with eosinophilic cytoplasm, vesicular nucleus, and prominent nucleoli. Septae were

Correspondence to: Dr. Ranjan Kumar Patel, Department of Radiodiagnosis, Maulana Azad Medical College, New Delhi - 110 002, India. E-mail: ranjanair1@gmail.com

(C) 2021 Creative Commons Attribution-NonCommercial 4.0 International License (CC BY-NC-ND 4.0). 


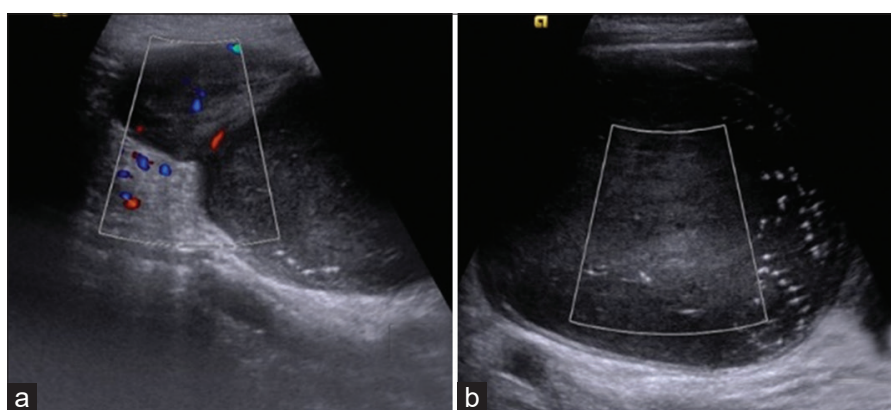

Figure 1: Transabdominal sonography reveals (a) a well-defined heterogeneous mass lesions with solid component showing vascularity on Doppler; (b) large necrotic component with multiple tiny echogenic foci

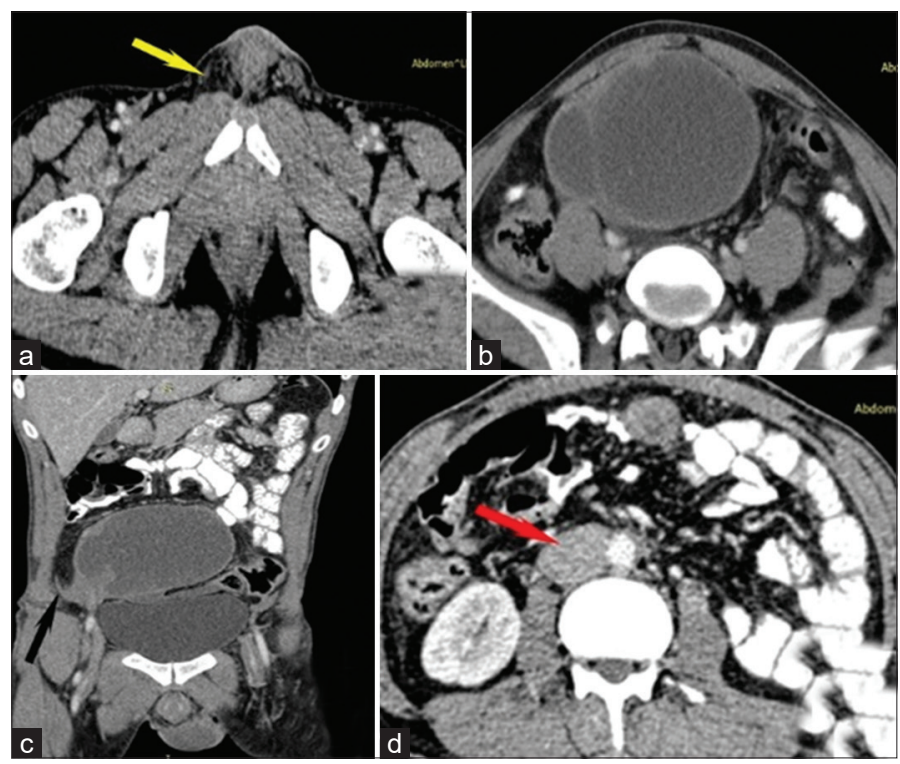

Figure 2: Contrast-enhanced computed tomography abdomen and pelvis reveals (a) absent right spermatic cord (yellow arrow) with (b) a well-circumscribed intra-abdominal mass lesion with enhancing solid areas and multiple necrotic non-enhancing components with focal peritoneal involvement (black arrow c). Enlarged metastatic aortocaval lymph node is seen in image $d$ (red arrow)
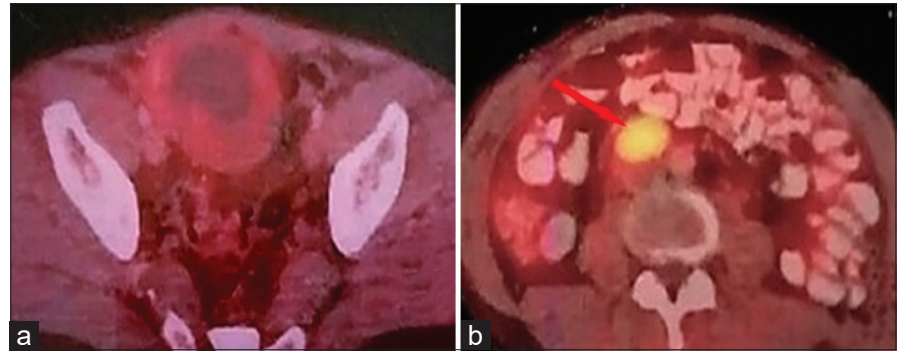

Figure 3: Axial fluorodeoxyglucose-positron emission tomographycomputed tomography image shows (a) FDG uptake in the peripheral solid component of tumor as well as (b) homogenous uptake in metastatic retroperitoneal node (red arrow)

infiltrated by lymphocytes. Multiple necrotic areas were seen. On immunohistochemistry, the cells were positive for placental alkaline phosphatase. Findings were suggestive of classical seminoma (Fig. 4).

The patient received post-operative chemotherapy that included three cycles of bleomycin, etoposide, and cisplatin
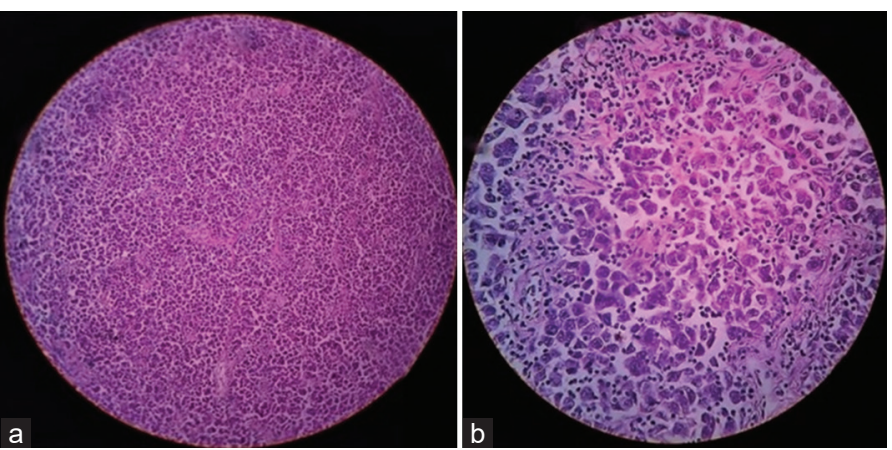

Figure 4: Histopathology (hematoxylin and eosin) reveals (a) tumor cells in sheets with intervening fibrous septae, infiltrated by lymphocytes $(\times 10$ view); (b) monomorphic population of cells showing scant to moderate amount of clear cytoplasm and central large nucleus and prominent nucleoli $(\times 40$ view $)$

regimen and two cycles of EP regimen (etoposide and cisplatin). At present, the patient is asymptomatic at 2-year post-operative follow-up.

\section{DISCUSSION}

Undescended testis is associated with various complications that include decreased fertility (bilateral cases), increased testicular germ cell tumors, testicular torsion, inguinal hernias, and psychological problems. The dreaded complication of cryptorchid testis is malignant transformation, highest in the intra-abdominal ectopic location [1]. Malignant transformation is most often seen in adult patients with its peak incidence in the third and fourth decades of life [3]. Although any histological variety can be seen, seminoma is the most common type [3]. In cryptorchid patients, normal contralateral testis also bears $5-20 \%$ risk of malignant transformation. Therefore, ultrasound screening of contralateral testis at regular intervals is advisable to detect early cancerous transformation [3].

Malignancies in the undescended testis are usually asymptomatic and are often detected incidentally on imaging [4]. Seminoma in the undescended testis can present as an abdominal wall tumor as reported by Yilmaz et al. [5]. CECT abdomen including the testicular region is the investigation of choice. The testicular region is often ignored while evaluating the CECT abdomen. Seminoma usually appears as a well-defined, homogeneous lesion, without obvious evidence of necrosis or calcification [6]. However, in our case, mass was heterogeneous owing to multiple necrotic areas as that of non-seminomatous tumors. It appears isointense on $\mathrm{T} 1 \mathrm{~W}$ images and hypointense to normal testicular parenchyma on T2W MR images [7]. The absence of a spermatic cord is an important imaging sign that indicates undescended testis. This sign is seen to be useful in diagnosing testicular torsion in an ectopic testis [8].

Another important diagnostic clue described by Lim et al. that may be helpful in diagnosing an intra-abdominal testicular tumor includes the identification of pampiniform plexus as multiple parallel veins and an ipsilateral draining vein that empties into the inferior vena cava (if right sided) or left renal vein (if left 
sided) [6]. It is a well-known fact that testicular germ cell tumors spread to retroperitoneal lymph nodes with choriocarcinoma being the exception that shows early hematogenous spread. Seminoma shows early metastasis to the retroperitoneal nodes before additional systemic involvement [9]. The present case also showed metastasis to aortocaval node. Peritoneal involvement is also not uncommon in case of intra-abdominal seminoma, as seen in our case [9].

Various tumor markers, such as beta-hCG, AFP, and LDH, play a crucial role in diagnosis, staging, prognosis, monitoring treatment response, and surveillance of testicular germ cell tumors. Pure seminoma does not produce AFP as seen in this case. Elevations of beta-hCG can also be seen in approximately $10-20 \%$ of patients with Stage I seminoma and up to $30-50 \%$ of cases of disseminated seminoma. LDH has limited sensitivity and specificity for seminoma, being elevated in $40-60 \%$ of men with testicular herm cell tumors. The higher the LDH levels, the more the tumor burden [10].

Surgical resection of the intra-abdominal tumor and enlarged lymph nodes is mandatory and chemotherapy may be an alternative, depending on the stage and histological variety of the lesion $[6,9]$.

\section{CONCLUSION}

Testicular malignancy should always be kept as a differential diagnosis of an intra-abdominal or retroperitoneal mass in a patient with a non-palpable testis. The testicular region should not be missed while evaluating an intra-abdominal mass in an adult male on imaging.

\section{REFERENCES}

1. Vikraman J, Hutson JM, Li R, Thorup J. The undescended testis: Clinical management and scientific advances. Semin Pediatr Surg 2016;25:241-8.

2. Fonkalsrud EW. Current management of undescended testis. Semin Pediatr Surg 1996;5:2-7.

3. Ferguson L, Agoulnik AI. Testicular cancer and cryptorchidism. Front Endocrinol 2013;4:1-5.

4. Miller FH, Whitney WS, Fitzgerald SW, Miller EI. Seminomas complicating undescended intraabdominal testes in patients with prior negative findings from surgical exploration. Am J Roentgenol 1999;172:425-8.

5. Yilmaz A, Bayraktar B, Sagiroglu J, Gucluer B. Giant seminoma in an undescended testis presenting as an abdominal wall mass. J Surg Case Rep 2011;2011:9-11.

6. Lim YJ, Jeong MJ, Bae BN, Kim SH, Kim JY. Seminoma in undescended testis. Abdom Imaging 2008;33:241-3.

7. Tsili AC, Tsampoulas C, Giannakopoulos X, Stefanou D, Alamanos Y, Sofikitis $\mathrm{N}$, et al. MRI in the histologic characterization of testicular neoplasms. Am J Roentgenol 2007;189:W331-7.

8. Geng JH, Huang CN. Torsion of undescended testis: Clinical, imaging, and surgical findings. Urol Sci 2014;25:31-4.

9. Moreno CC, Small WC, Camacho JC, Master V, Kokabi N, Lewis M, et al. Testicular tumors: What radiologists need to know differential diagnosis, staging, and management. Radiographics 2015;35:400-15.

10. Gilligan TD, Seidenfeld J, Basch EM, Einhorn LH, Fancher T, Smith DC, et al. American society of clinical oncology clinical practice guideline on uses of serum tumor markers in adult male with germ cell tumors. J Clin Oncol 2010;28:3388-404.

\section{Funding: None; Conflicts of Interest: None Stated.}

How to cite this article: Patel RK, Sarda H. Intra-abdominal seminoma in undescended testis with retroperitoneal nodal metastasis: A case report. Indian J Case Reports. 2021;7(4):167-169. 